\title{
Fusion of primary teeth- An odontogenic anomaly
}

\author{
Puneet Kumar $^{1, *}$, Jasmine Kaur ${ }^{2}$, Kanika Gupta Verma ${ }^{3}$, Virinder Goyal ${ }^{4}$, Suruchi Juneja ${ }^{5}$ \\ ${ }^{1,2}$ PG Student, ${ }^{3,5}$ Reader, ${ }^{4}$ Professor \& HOD, Dept. of Pedodontics, Surendera Dental College and Research Institute, \\ Ganganagar, Rajasthan, India
}

*Corresponding Author:

Email: puneetgdr01@gmail.com

\begin{abstract}
The conjoining or twinning of teeth leads to odontogenic anomalies. The dental fusion is observed by the union of two normally separated tooth germs. It is defined as the union of two independently developing primary or permanent teeth that results in the formation of a single large tooth. It can be complete or incomplete, depending upon the stage of development of teeth at the time of union. The prevalence of this anomaly is $<1 \%$ in primary dentition and is commonly observed in incisor-canine region.
\end{abstract}

Keywords: Fusion, Primary dentition, Unilateral.

\section{Introduction}

Gemination and fusion are commonly observed dental anomalies occurring in primary and permanent dentition; also known as "double tooth", "joined teeth" or "fused teeth". ${ }^{1}$ The union of two normally separated tooth germs results in a rare complex developmental anomaly called Fusion. The fused tooth may be normal or larger in size. Fusion can be observed between the primary or permanent teeth and may involve supernumerary teeth. ${ }^{2}$ Fusion is more commonly seen in primary dentition than permanent. The prevalence of fusion in the primary dentition for unilateral presentation is $0.1 \%$ to $1.5 \%$ with no sex predilection, whereas in Indian population Reddy and Munshi found $0.14 \%$ prevalence..$^{2-6}$

Various etiological factors causing fusion can be trauma, heredity, environmental factors and genetics. ${ }^{7}$ the occurence of fused teeth is commonly observed in incisor and canine region, which can be unilateral or bilateral involving maxillary or mandibular arches. ${ }^{8}$ Various clinical problems observed with fused teeth are esthetics, spacing, periodontal problems etc. Fused primary teeth may also be associated with the agenesis of permanent teeth. It also can give rise to malalignment and increased incident of dental caries. ${ }^{1}$

\section{Case report}

A 8 year old boy reported to the Department of Pediatric and Preventive Dentistry, Surendera Dental College and Research Institute, Sriganganagar with the chief complaint of pain in lower right back tooth region. Pain was dull, continuous and non-radiating. No history of sensitivity to hot or cold. No relevant past medical and dental history was found. On soft tissue examination, gingiva was reddish pink in color with rolled out margins and bleeding on probing was present. On hard tissue examination, type of dentition seen was mixed (Fig. 1 and 2), faulty GIC restorations were present wrt 36, 84, 85, 46 and dental caries wrt 54, 55 and 75 . On clinical examination, mandibular right primary lateral incisor and primary canine were joined together, separated with a groove in between both crown structures. On radiographic examination (Fig. 3), the union between the two teeth was observed to be complete with radicular portion at cementum; with two separate root canals. Orthopantomograph (Fig. 4) revealed no abnormality except congenital missing 41 . As this odontogenic anomaly was observed as evident finding during examination and patient was having no complaint, thus no intervention was planned. The treatment planned was GIC restorations wrt 54, 55 and 75 , composite restorations wrt 36 and 46, pulpectomy followed stainless steel crowns wrt 84 and 85, extraction wrt 74 and prosthodontic consideration wrt 41 , to maintain form and function of dentition.

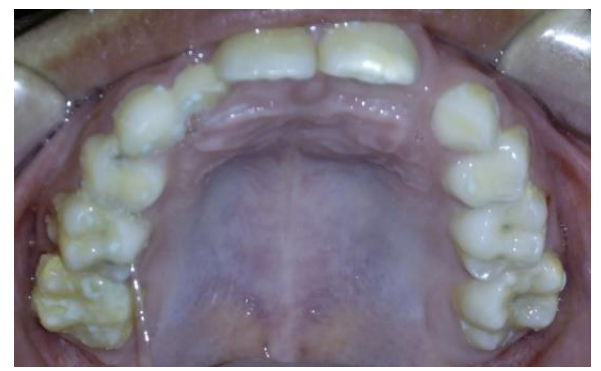

Fig. 1: Maxillary arch view showing dental caries wr 54 and 65

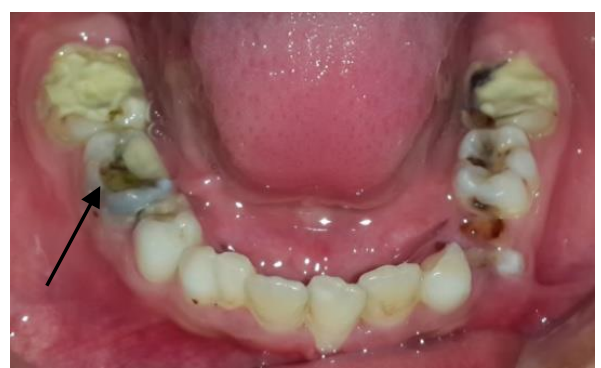

Fig. 2: Mandibular arch view showing fusion wrt 82 83 , separated with groove 


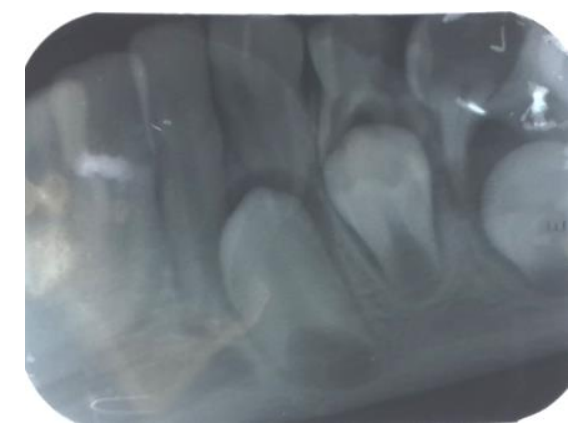

Fig. 3: IOPA wrt 8283 showing fusion of the roots with two separate root canals

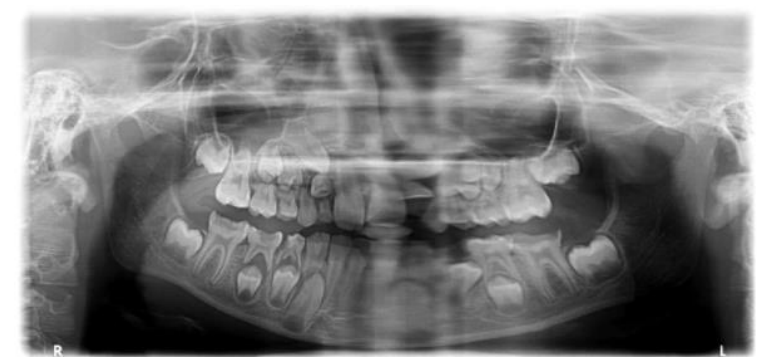

Fig. 4: Orthopantomograph showing missing 41

\section{Discussion}

Fusion is an odontogenic anomaly associated with union of two normally separated tooth germs. It can be complete or incomplete depending upon time of union during developmental stage of tooth development. ${ }^{2}$ Although etiology of fused teeth is unknown, but some researchers proposed that physical forces could bring the developing teeth in contact, causing necrosis of the epithelial tissue (which separates them), resulting in fusion of teeth. Other theory suggests that the fusion results from embryological persistence of the inter-dental lamina between two germs. ${ }^{1}$

The other dental anomaly associated with number of teeth is gemination, which represents division of a single tooth germ by invagination, resulting in incomplete formation of two teeth. In the present case, the primary mandibular teeth i.e., 82 and 83 showing fusion are succeeded by normally developing successors.

The clinical appearance of a fused tooth is relatively broad and shows either a bifid crown appearance with a groove delineating the two crowns or an incisal notch. The groove may continue onto the root if they are also conjoined, but maxillary fused teeth usually show two separate roots. Fused teeth may involve two separate or conjoined pulp chambers, with two fused root canals or two independent endodontic systems. ${ }^{1,9}$ In the present case, a groove was noticed between the crowns of 82 and 83 with complete fusion of crowns and roots but having two separate root canals.

Clinical problems that encountered are unaesthetic appearance, malocclusion, and susceptibility to dental caries. ${ }^{2}$ Due to increased root surface area of fused tooth, relative to the size of permanent successor crown, the root resorption of fused tooth is delayed causing retention of tooth for prolonged duration, leading to impacted permanent successor and malocclusion. ${ }^{4}$ Microdontia and delayed tooth formation is also observed with fused primary teeth. Thus, it is important to monitor the clinical situation of permanent dentition during treatment planning for management of fusion. ${ }^{2,4}$

In pediatric patients, management depends upon level of fusion, teeth involved and cooperation of patient. It involves retaining the fused teeth in oral cavity till the time of exfoliation, with proper oral hygiene instructions. If fused tooth is carious, the restoration is preferred option and if pulpally involved, endodontic management can be done. To ensure predictable results of endodontic management, advanced imaging techniques like cone beam computed tomography can be used for diagnosis and treatment planning. ${ }^{2}$

The extraction of tooth is planned after observing the permanent successor, ensuring the esthetics and functional occlusion. In the present case, the preventive approach was planned till the physiological exfoliation of fused tooth and eruption of its successor i.e right permanent mandibular canine.

\section{Conclusion}

During routine clinical examination, the fused teeth are frequently observed. Thus, understanding the prevalence of fused teeth and a thorough clinical and radiographic evaluation is essential for proper diagnosis and treatment planning. These anomalies may influence tooth alignment, symmetry, interdigitation, esthetics and periodontal conditions. Hence, it is necessary to recognize this dental anomaly at the earliest, with appropriate treatment plan such as restoration, endodontic, prosthodontic and orthodontic consideration according to patients expectation and degree of compliance.

\section{References}

1. Eregowda N I, Pathak S, Anitha SR, Poornima P. Unilateral Double Teeth in Primary Dentition. Univ Res J Dent 2015;5(3):198-9.

2. Mehta M. Fusion of Primary Mandibular Anterior Teeth Associated with Partial Anodontia of Primary and Permanent Dentition: A Case Report. J Dent Health Oral Disord Ther 2015;3(3):1-3.

3. Shafer, Hine, Levy. Shafer's Textbook of Oral Pathology. 7th ed. India: Elsevier; 2012. pp. 41.

4. Ahmet ES, Yildiray S, Yasin Y, Halil S, Abdullah E. Prevalence of fusion and gemination in permanent teeth in Coppadocia region in Turkey. Pak Oral Dent $\mathrm{J}$ 2011;31(1):17-22.

5. Grahnen $\mathrm{H}$ and Granath LE. Numerical variations in primary dentition and their correlation with the permanent dentition. Odontol Revy 1961;4:348-57.

6. Reddy NN and Munshi AK. Fusion of primary incisors: A report of six cases. J Indian Soc Pedod Prev Dent 1999;17(2): 55-60. 
7. Hagman FT. Anomalies of form and number, fused primary teeth, a correlation of the dentitions. ACDS J Dent Child 1988;55(5): 359-61.

8. Wu CW, Lin YT, Lin YT. Double primary teeth in children under 17 years old and their correlation with permanent successors. Chang Gung Med J 2010;33(2):188-93.

9. Penumatsa NV, Nallanchakrava S, Dandempally A. Fusion of mandibular primary incisors and their permanent successors: A case report with review of literature. J Oral Res Rev 2012;4(2):62-6. 